\title{
ÍNDICE DE APGAR CORRELACIONADO A FATORES MATERNOS, OBSTÉTRICOS E NEONATAIS A PARTIR DE DADOS COLETADOS NO CENTRO DE SAÚDE DA FAMÍLIA DO BAIRRO DOM EXPEDITO LOPES SITUADO NO MUNICÍPIO DE SOBRAL/CE
}

\author{
APGAR SCORE CORRELATED TO MATERNAL AND OBSTETRIC FACTORS FROM DATA \\ COLLECTED AT THE FAMILY HEALTH CENTER OF THE DOM EXPEDITO LOPES \\ NEIGHBORHOOD LOCATED IN THE MUNICIPALITY OF SOBRAL / CE
}

Leonardo Sales Ribeiro Silva', Ana Neiline Cavalcante², José Klauber Roger Carneiro², Maria Auxiliadora Silva Oliveira ${ }^{2}$

1 - Acadêmica do curso de Medicina do Centro Universitário Inta (UNINTA), bolsista do Programa de Bolsas de Iniciação Científica UNINTA, 2 - Docente do curso de Medicina do Centro Universitário Inta (UNINTA), rua Antônio Rodrigues Magalhães, Dom Expedito, Sobral/CE

\section{RESUMO}

OBJETIVO: Analisar a influência dos fatores Maternos, obstétricos e neonatais no resultado da avaliação do Índice de Apgar de neonatos no Centro de Saúde da Família do bairro Dom Expedito Lopes na cidade de Sobral/CE. MÉTODOS: Trata-se de uma pesquisa retrospectiva, quantitativa e descritiva, tendo como fonte de dados os prontuários de gestantes atendidas nos anos de 2016 e 2017. As variáveis analisadas foram aquelas que permitissem correlacionar o Índice de Apgar com os fatores maternos, obstétricos e neonatais: idade materna, tipo de parto, número de consultas pré-natal, idade gestacional e peso ao nascer. $O$ presente trabalho foi submetido ao comitê de ética local tendo sido aprovado com número de parecer 1.878.614, manteve o anonimato e seguiu as recomendações da Portaria do Conselho Nacional de Saúde/MS - CNS, Resolução 466/12, adotando os quatro princípios básicos da bioética: autonomia, beneficência, não maleficência e justiça. RESULTADOS: Na maioria das gestantes o percentual da faixa etária foi dos 21 aos 30 anos $(56,25 \%)$ com índice de Apgar satisfatório, ou seja, acima de sete. Em relação ao tipo de parto pode-se constatar que o parto Cesário esteve bem presente com $62,5 \%$ com escore de 7-10. Sobre o número de consultas pré-natal a maioria realizou 07 ou mais consultas $(82,81 \%)$ com escore Apgar acima de 7. Para a Idade gestacional a maioria esteve entre 37 a 41 semanas, sendo considerada a termo e que obtiveram o peso ao nascer maior ou igual a $2.500 \mathrm{~g}$, apresentando Apgar acima de 7. CONCLUSÃO: Estes achados possibilitam visualizar a importância e a adesão das gestantes à um acompanhamento de pré-natal de qualidade, resultando numa redução na mortalidade neonatal e um índice de Apgar satisfatório.

Descritores: Índice de Apgar, Gestante, Pré-natal.

\section{ABSTRACT}

OBJECTIVE: To analyze the influence of maternal, obstetrical and neonatal factors on the outcome of the evaluation of the Apgar Index of newborns at the Family Health Center in the neighborhood of Dom Expedito Lopes in the city of Sobral/CE. METHODS: It is a retrospective, quantitative and descriptive study, having as a data source the charts of pregnant women attended in the years 2016 and 2017. The variables analyzed were those that allowed to correlate the Apgar Index with maternal, obstetric and neonatal factors: maternal age, type of delivery, number of prenatal consultations, gestational age and birth weight. The present work was submitted to the local ethics committee and approved with the number of opinion 1.878.614, it remained anonymous and followed the recommendations of the Ordinance of the National Health Council/MS - CNS, Resolution 466/12, adopting the four basic principles of bioethics: autonomy, beneficence, nonmaleficence and justice. RESULTS: In the majority of pregnant women, the percentage of the age group was from 21 to 30 years old (56.25\%) with a satisfactory Apgar score, that is, above seven. Regarding the type of delivery, it can be seen that the Cesário delivery was well present with $62.5 \%$ with a score of 7-10. Regarding the number of prenatal consultations, the majority performed 07 or more consultations (82.81\%) with an Apgar score above 7. For gestational age the majority were between 37 and 41 weeks, being considered at term and that they obtained the weight at birth greater than or equal to 2,500g, presenting Apgar above 7. CONCLUSION: These findings make it possible to visualize the importance and adherence of pregnant women to a quality prenatal follow-up, resulting in a reduction in neonatal mortality and a satisfactory Apgar score.

Descriptors: Apgar score, Pregnant woman, Prenatal care. 


\section{INTRODUÇÃO}

As taxas de morbidade e mortalidade neonatal abrangem condições além das questões de vida e saúde da população, pois podem também mensurar o nível de desenvolvimento socioeconômico de um país. A mortalidade neonatal foi alvo de discussão em estudos que refletiam a respeito dos Objetivos de Desenvolvimento do Milênio, uma vez que representa parcela significativa da mortalidade infantil, relevante indicador de saúde. No Brasil, a cada dois óbitos em menores de um ano de idade, pelo menos um acontecia nos primeiros seis dias de vida, o que evidencia a importância de estratégias de fortalecimento das políticas voltadas para assistência ao pré-natal, parto e recém-nascido (1).

O Índice de Apgar foi descrito em 1952 pela anestesista americana Virgínea de Apgar. Este Índice toma proporções úteis para a garantia da avaliação inicial ao neonato, considerando, para tanto, valores baixos no primeiro e quinto minutos de avaliação um risco de morte neonatal (2).

Após o nascimento, o bebê é avaliado pelo pediatra, sendo ele o responsável por informar o Apgar do recém-nascido no 1 을 minuto e no final do 5응 minuto. As notas da escala de Apgar são baseadas na cor, na respiração, na frequência cardíaca, no tônus muscular e na resposta do recém-nascido a estímulos por sonda. Cada um desses cinco aspectos recebe notas que vão de 0 a 2 e que, ao serem somadas, podem totalizar um valor que vai de 0 a 10, sendo que resultados maiores que sete são considerados adequados (3).

Um escore de Apgar de 7-10 denota uma criança sadia, que possivelmente não terá problemas futuros. Quando $<7$, é sinal de alerta para uma atenção maior (4).

Por ser a única forma de avaliação em países em desenvolvimento, onde os exames laboratoriais podem não estar disponíveis, o baixo valor do escore de Apgar é útil para identificar as crianças que necessitam de cuidados adicionais, mesmo na ausência de dados laboratoriais. Existe consenso de que um escore de Apgar de 7-10 significa uma criança sadia, que provavelmente não terá problemas futuros. Quando inferior a 7, é sinal de alerta para atenção especial. Há diferentes níveis de escore de Apgar baixo, de acordo com alterações fisiopatológicas. Além disso, é parcialmente dependente da maturidade do concepto (5).

Conhecer os fatores associados ao baixo
Apgar, são de suma importância, pois servirão de subsídio para que sejam empreendidos esforços por parte dos serviços de saúde a populações que apresentem estas características (6).

Portanto, o presente estudo tem como objetivo principal analisar a influência dos fatores maternos e obstétricos no resultado da avaliação do Índice de Apgar de neonatos no Centro de Saúde da Família do bairro Dom Expedito Lopes na cidade de Sobral/CE.

\section{METODOLOGIA}

A pesquisa foi realizada no Centro de Saúde da Família do bairro Dom Expedito Lopes situado no município de Sobral/CE.

Trata-se de um estudo de caráter exploratório, quantitativo, descritivo, retrospectivo e com análise documental.

Os sujeitos da pesquisa foram os recémnascidos $(n=64)$ atendidas no referido Centro de Saúde da Família, cujas fichas de acompanhamento e/ou prontuários datassem do ano de 2016 e 2017. Foram excluídas as fichas e/ou prontuários de anos diferentes deste tempo pré-estabelecido.

As variáveis analisadas foram aquelas que permitissem correlacionar o Índice de Apgar com os fatores maternos, obstétricos e neonatais: idade materna, tipo de parto, número de consultas prénatal, idade gestacional e peso ao nascer.

Os dados foram coletados a partir da fichas/prontuários de acompanhamento das gestantes que foram atendidas no Centro de Saúde da Família. Os dados foram analisados em Excell e foram confeccionadas tabelas contendo frequências absolutas e relativas.

O presente trabalho foi submetido ao comitê de ética local tendo sido aprovado com número de parecer 1.878.614, manteve o anonimato e seguiu as recomendações da Portaria do Conselho Nacional de Saúde/MS - CNS, Resolução 466/12, adotando os quatro princípios básicos da bioética: autonomia, beneficência, não maleficência e justiça.

\section{RESULTADOS}

A tabela a seguir trata da relação índice de Apgar com o número total encontrado do seu respectivo valor índice.

Como pode ser observado na tabela 2 , a relação da idade materna com os seus respectivos valores encontrados no Índice de Apgar.

Encontra-se na tabela 03 a relação do tipo de 
[ÍNDICE DE APGAR CORRELACIONADO A FATORES MATERNOS, OBSTÉTRICOS E NEONATAIS A PARTIR DE DADOS COLETADOS NO CENTRO DE SAÚDE DA FAMÍLIA DO BAIRRO DOM EXPEDITO LOPES SITUADO NO MUNICÍPIO DE SOBRAL/CE ] - Silva L.S.R. et al

Tabela 01: Índice de Apgar dos recém-nascidos vivos registrados em prontuários no Centro de Saúde da Família do bairro Dom Expedito Lopes situado no município de Sobral/CE aos primeiros cinco minutos $\left(5^{\prime}\right)$.

\begin{tabular}{c|c|c}
\hline Índice de Apgar aos 5' & $\mathbf{n}$ & $\%$ \\
\hline $0-3$ & 1 & 1,56 \\
\hline $4-6$ & 0 & 0,00 \\
$7-10$ & 63 & 98,43 \\
\hline
\end{tabular}

Fonte: dados da pesquisa.

Tabela 02 - Distribuição da faixa etária de gestantes atendidas no Centro de Saúde da Família do bairro Dom Expedito Lopes situado no município de Sobral/CE correlacionado ao Índice de Apgar nos primeiros cinco minutos $\left(5^{\prime}\right)$.

\begin{tabular}{|c|c|c|c|c|c|c|}
\hline \multirow{2}{*}{$\begin{array}{l}\text { Idade } \\
\text { Materna }\end{array}$} & \multicolumn{5}{|c|}{ Índice de Apgar aos 5' } \\
\cline { 2 - 8 } (anos) & \multicolumn{2}{|c|}{$\mathbf{0 - 3}$} & \multicolumn{2}{|c|}{$4-6$} & \multicolumn{2}{|c|}{$7-10$} \\
\cline { 2 - 8 } & $\mathrm{n}$ & $\%$ & $\mathrm{n}$ & $\%$ & $\mathrm{~N}$ & $\%$ \\
\hline De 12 a 20 & 00 & 0,00 & 00 & 0,00 & 13 & 20,31 \\
\hline De 21 a 30 & 01 & 1,56 & 00 & 0,00 & 36 & 56,25 \\
\hline De 31 a 40 & 00 & 0,00 & 00 & 0,00 & 12 & 18,75 \\
\hline De 41 & 00 & 0,00 & 00 & 0,00 & 02 & 3,12 \\
\hline
\end{tabular}

Fonte: dados da pesquisa.

Tabela 03 - Tipo de parto correlacionado ao Índice de Apgar nos primeiros cinco minutos (5') registrado em prontuários do Centro de Saúde da Família do bairro Dom Expedito Lopes situado no município de Sobral/CE.

\begin{tabular}{|l|c|c|c|c|c|c|}
\hline \multirow{2}{*}{$\begin{array}{l}\text { Tipo de } \\
\text { parto }\end{array}$} & \multicolumn{6}{|c|}{ Índice de Apgar aos 5' } \\
\cline { 2 - 8 } & \multicolumn{2}{|c|}{$\mathbf{0 - 3}$} & \multicolumn{2}{|c|}{$4-6$} & \multicolumn{2}{c|}{$7-10$} \\
\cline { 2 - 8 } & $\mathbf{n}$ & $\%$ & $n$ & $\%$ & $n$ & $\%$ \\
\hline Cesária & 00 & 0,00 & 00 & 0,00 & 40 & 62,5 \\
\hline Vaginal & 01 & 1,56 & 00 & 0,00 & 23 & 35,93 \\
\hline
\end{tabular}

parto, cesáreo ou vaginal, com os valores encontrados do Índice de Apgar.

$\mathrm{Na}$ tabela 04 a seguir é mostrada a relação do número de consultas realizadas durante o prénatal e o Índice de Apgar respectivo.

A tabela 05 demostra a relação da idade gestacional

Tabela 04 - Número de consultas pré-natais realizadas em gestantes atendidas no Centro de Saúde da Família do bairro Dom Expedito Lopes situado no município de Sobral/CE correlacionado aos Índices de Apgar nos primeiros cinco minutos ( $\left.5^{\prime}\right)$.

\begin{tabular}{|c|c|c|c|c|c|c|}
\hline \multirow{3}{*}{$\begin{array}{l}\text { Número de } \\
\text { consultas } \\
\text { pré-natais }\end{array}$} & \multicolumn{6}{|c|}{ Índice de Apgar aos 5' } \\
\hline & \multicolumn{2}{|c|}{$0-3$} & \multicolumn{2}{|c|}{ 4-6 } & \multicolumn{2}{|c|}{$7-10$} \\
\hline & $\mathrm{n}$ & $\%$ & $\mathrm{n}$ & $\%$ & $\mathbf{N}$ & $\%$ \\
\hline De 1 a 3 & 00 & 0,00 & 00 & 0,00 & 00 & 0,00 \\
\hline De 4 a 6 & 00 & 0,00 & 00 & 0,00 & 10 & 15,62 \\
\hline$\geq 7$ & 01 & 1,56 & 00 & 0,00 & 53 & 82,81 \\
\hline
\end{tabular}

Fonte: dados da pesquisa. com Índice de Apgar nos primeiros cinco minutos (5'). Observa-se na tabela a seguir a relação do Índice de Apgar com o peso ao nascer.

Tabela 05 - Idade gestacional (duração da gestação em semanas) em gestantes atendidas no Centro de Saúde da Família do bairro Dom Expedito Lopes situado no município de Sobral/CE frente aos Índices de Apgar nos primeiros cinco minutos (5').

\begin{tabular}{|c|c|c|c|c|c|c|}
\hline \multirow{3}{*}{$\begin{array}{l}\text { Idade } \\
\text { Gestacional } \\
\text { (semanas) }\end{array}$} & \multicolumn{6}{|c|}{ Índice de Apgar aos 5' } \\
\hline & \multicolumn{2}{|c|}{$0-3$} & \multicolumn{2}{|c|}{ 4-6 } & \multicolumn{2}{|c|}{$7-10$} \\
\hline & n & $\%$ & $n$ & $\%$ & $n$ & $\%$ \\
\hline De 22 a 27 & 00 & 0,00 & 00 & 0,00 & 01 & 1,56 \\
\hline De 28 a 36 & 00 & 0,00 & 00 & 0,00 & 13 & 20,31 \\
\hline De 37 a 41 & 01 & 1,56 & 00 & 0,00 & 45 & 70,31 \\
\hline$>42$ & 00 & 0,00 & 00 & 0,00 & 04 & 6,25 \\
\hline
\end{tabular}

\section{DISCUSSÃO}

A tabela 01 mostra que $98,43 \%$ obtiveram um excelente Índice de Apgar na faixa de classificação entre 7 e 10 nos primeiros 05 minutos de vida, ou seja, denota uma criança sadia (7). Para o intervalo de 4 a 6 , sinal de alerta para atenção especial, não foi observado nenhum recém-nascido (8).

Foi observado, também, que 1,56\% obtiveram um baixo Índice de Apgar, entre 0 e 3 . A literatura relata que baixo índice de Apgar implica maior risco de mortalidade neonatal (9).

O índice de Apgar, como salientado, é um importante indicador de risco para a morbimortalidade perinatal. Estudo realizado para o Município de São Paulo em 1998 considerou que o baixo índice de Apgar é um bom indicador de morbidade e está associado à mortalidade neonatal; outro estudo, desenvolvido para comparar condições sócio-econômicas e características neonatais no Rio de Janeiro em 1994, também concluiu que o índice de Apgar é um importante indicador de risco; finalmente, de acordo com estudo realizado no Hospital Universitário de Dakar entre 1994 e 2003, a mortalidade neonatal precoce atinge particularmente os nascidos vivos com baixo índice de Apgar (10).

Em consequência, a mortalidade neonatal, pode estar relacionada a variáveis diversas (5). Entre essas, podemos incluir a idade materna (tabela 2), o tipo de parto (tabela 3), o número de consultas (tabela 4), a idade gestacional (IG) (tabela 5) e o peso ao nascer (tabela 6).

$\mathrm{Na}$ tabela 02 observa-se que na maioria das gestantes o percentual da faixa etária foi dos 21 aos 
30 anos (56,25\%) com índice de Apgar satisfatório, ou seja, acima de sete, enquanto que 1 gestantes apresentou um escore menor que 7 .

Tabela 06 - Peso dos recém-nascidos no Centro de Saúde da Família do bairro Dom Expedito Lopes situado no município de Sobral/CE correlacionado ao Índice de Apgar nos primeiros cinco minutos $\left(5^{\prime}\right)$.

\begin{tabular}{|c|c|c|c|c|c|c|}
\hline \multirow{3}{*}{$\begin{array}{l}\text { Peso ao } \\
\text { Nascer }\end{array}$} & \multicolumn{6}{|c|}{ Índice de Apgar aos 5' } \\
\hline & \multicolumn{2}{|c|}{$0-3$} & \multicolumn{2}{|c|}{ 4-6 } & \multicolumn{2}{|c|}{$7-10$} \\
\hline & $n$ & $\%$ & $n$ & $\%$ & $n$ & $\%$ \\
\hline$<2.500 \mathrm{~g}$ & 01 & 1,56 & 00 & 0,00 & 10 & 15,62 \\
\hline$=2.500 \mathrm{~g}$ & 00 & 0,00 & 00 & 0,00 & 53 & 82,81 \\
\hline
\end{tabular}

Em relação ao Índice de Apgar, no estudo realizado por Ximenes e Oliveira a porcentagem de recém-nascidos com Apgar considerado ideal (7-10) foi maior nas mulheres entre 20 e 35 anos (82\%) (11). Com efeito, em relação a idade materna superior a 40 anos foi percebido um declínio nessa faixa do Índice de Apgar, entre 07 e 10. A gravidez após a idade de 34 anos é denominada gravidez tardia, sendo considerada fator de risco para a morbidade materna e fetal (12).

A literatura cita uma frequência cerca de duas vezes maior de morte neonatal e quatro vezes maior de morte fetal tardia entra as gestantes com 40 anos ou mais (13).

No tipo de parto pode-se constatar que o parto Cesário esteve bem presente com 62,5\% com escore de 7-10.

A correlação do tipo de parto com a vitalidade do recém-nascido, avaliado com o índice de Apgar, não obteve resultados significativos devido ao fato que os recém nascidos obtiveram um índice de Apgar satisfatório, ou seja, acima de sete, tanto no parto normal, quanto no parto cesariano. Daqueles que nasceram de parto cesáreo, 150 $(98,0 \%)$ tiveram o índice de Apgar satisfatório no 1 을 minuto, enquanto o de parto normal foi de 145 $(98,6 \%)$. No quinto minuto todos tiveram o índice de Apgar satisfatório, independente do tipo de parto. Na pesquisa de Kilsztajn et al. (2007) (10) foi obtido a mesma conclusão, pelo mesmo motivo, os que obtiveram um índice de Apgar satisfatório, foram $99,3 \%$ e $99,2 \%$ no parto cesáreo e normal respectivamente (3).

Apesar do avanço técnico nas práticas cirúrgicas, a morbimortalidade materna continua associada ao parto cesáreo, os partos cirúrgicos só deveriam ser empregados quando rigorosamente indicados. Contudo, no processo de medicalização da gestação, as cesáreas são hoje consideradas no Brasil a forma moderna e conveniente de parto. Os médicos têm preferido os partos cesáreos porque são mais rápidos que os morosos partos vaginais e utilizam os motivos manifestos por um pequeno número de mulheres que demanda partos cesáreos para generalizar a suposta preferência das gestantes pela cesariana (10).

Em relação às características assistenciais pode-se citar o número de consulta pré-natal e Apgar no $1^{\circ}$ e $5^{\circ}$ minuto como fatores de risco e por isso determinantes para a mortalidade neonatal. Garantido à gestante um acompanhamento pré-natal de qualidade, com número adequado de consultas, assistência apropriada durante o parto, ao recémnascido e a puérpera poderíamos ter uma redução na mortalidade neonatal (14).

De acordo com a análise dos dados acima, sobre o número de consultas pré-natal a maioria realizou 07 ou mais consultas $(82,81 \%)$ com escore Apgar acima de 7.

O número mínimo preconizado pelo Ministério da Saúde para todas as gestantes é de seis consultas, com início o mais precocemente possível, sendo assim distribuídas: uma no 10 trimestre (até a 12a semana), duas no 2 으 trimestre e três no 3 응 trimestre; considerando o risco perinatal e as intercorrências clínico-obstétricas, mais comuns no 3o trimestre, é imprescindível que sejam realizadas consultas até a 40a semana gestação; gestantes cujo trabalho de parto não se inicie até a 41 1 devem ser encaminhadas à maternidade de referência para a interrupção da gravidez (15).

Durante período investigado foram encontrado em sua maioria recém-nascido à termo (37 a 41 semanas) e nesse intervalo, apenas um com Índice de Apgar nos primeiros cinco minutos menor que 7.

A morbilidade e a mortalidade perinatal estiveram sempre associadas com parto pré-termo, pós-termo e restrição de crescimento intra-uterino. 0 conhecimento preciso da idade gestacional e da data provável do parto é, por isso, fundamental em Medicina Perinatal, para a correta orientação obstétrica e definição de estratégias assistenciais (16).

Avaliando a idade gestacional entre 37 a 41 semanas e o Apgar do recém-nascido no $5^{\circ}$ minuto foi observado que $100 \%$ apresentaram Apgar entre 7 e 10. Concordantemente com as informações das declarações de nascimento processadas pela 
Fundação Sistema Estadual de Análise de Dados (Fundação SEADE) no estado de São Paulo em 2003, revelou que de 469.015 gestantes, com idade gestacional entre 37 a 41 semanas, 99,3\% apresentaram Apgar no $5^{\circ}$ minuto entre 7 e 10, enquanto $0,67 \%$ apresentaram Apgar no $5^{\circ}$ minuto entre 0 e 6 , demostrando assim que uma idade gestacional adequada favorece a melhor pontuação pela avaliação do Apgar (3).

$\mathrm{Na}$ tabela 06 observa-se que o peso ao nascer maior ou igual a $2.500 \mathrm{~g}$ obteve um intervalo de Apgar alto entre 7 e 10, correspondendo a cerca de $82,81 \%$. Enquanto o peso ao nascer abaixo de $2.500 \mathrm{~g}$ representou aproximadamente $15,62 \%$ de acordo com os dados coletados e 1 RN com Índice de Apgar menor ou igual a 3.

A Organização Mundial da Saúde (OMS) define como baixo peso ao nascer (BPN) o nascimento de recém-nascidos com peso inferior a $2.500 \mathrm{~g}$. Os principais responsáveis por esta condição são o retardo do crescimento intra-uterino (RCIU) e o período gestacional curto, ou seja, a prematuridade (17).
O baixo valor de Apgar aos 5' e o baixo peso ao nascer são os mais expressivos fatores de risco para a mortalidade neonatal. Existe, de certo, uma preocupação geral com a intensificação de investimentos na prevenção do baixo peso ao nascer, provocada pelas evidências de sua alta incidência no país (18).

\section{CONCLUSÃO}

Os escores de Apgar é uma medida do bemestar do recém-nascido e útil para identificar as crianças que necessitam de cuidados adicionais, mesmo na ausência de dados laboratoriais. Os resultados da pesquisa relacionando o Apgar com a idade materna, o tipo de parto, o número de consultas, a idade gestacional e o peso ao nascer demonstraram o índice Apgar no final do 5o minuto alto em suagrande maioria entre 7 e 10.

Estes achados possibilitam visualizar a importância do acompanhamento pré-natal de qualidade, resultando numa diminuição da mortalidade neonatal e um índice de Apgar satisfatório.

\section{REFERÊNCIAS:}

1- Saraiva JP, Vogt SE, Simão DAS, Duarte ED, Rocha JS. Associação entre fatores maternos e neonatais e o Apgar em recém-nascidos de risco habitual. Rev Rene. 2018, 19: 3179-3179. Disponível:

http://periodicos.ufc.br/rene/article/download/31404/pdf

2- Zorzi PM, Madi SRC, Rombaldi RL, Araújo BF, Zatti H, Madi JM, Barazzetti DO. Fatores Perinatais Associados a RecémNascidos de Termo com pH<7,1 na Artéria Umbilical e Índice de Apgar $<7,0$ no 5 Minuto. Rev Bras Ginecol Obstetríc. 2012, 34 (8):381-385. Disponível: http://www.scielo.br/pdf/rbgo/v34n8/07.pdf

3- Aguiar OO, Ferreira OC, Filho IP, Jabbar MS, Pereira ACA, Shimabuco LK, Shinzato TH. A importância do pré-natal parao nascimento saudável em uma maternidade de Campo Grande-MS. Ensaios Ciência: Ciências Biológicas, Agrárias e da Saúde. 2013, 17(3).Disponível:http://www.pgsskroton.com.br/seer/index.php/ensaioeciencia/article/view/2325/2228 4- Silva HTFML, Tavares FM. Perfil gestacional, obstétrico e neonatal na unidade de terapia intensiva de um hospital público do interior da Bahia. Rev Saúde.Com. 2017, 13(3). Disponível:

http://periodicos2.uesb.br/index.php/rsc/article/download/3322/2762/

5- Oliveira TG, Arrelaro RC, Freire PV, Moraes JS, Moreira FT, Ricardi VA, Rossi S, Juliano Y et al. Escore de Apgar e mortalidade neonatal em um hospital localizado na zona sul do município de São Paulo. Einstein, 2012, 10(1). Disponível: http://www.scielo.br/pdf/eins/v10n1/pt_v10n1a06.pdf

6- Prado V, Fontes KB, Schmidt KT. Fatores associados a vitalidade ao nascer. Arq Ciênc Saúde UNIPAR. 2015,19(1).

Disponível:http://revistas.unipar.br/index.php/saude/article/viewFile/5263/3036

7- AMIB Associação de Medicina Intensiva Brasileira. Recomendações para o Reconhecimento e Abordagem do Recém-

nascido, da Criança e do Adolescente com Doença Grave causada pelo vírus Influenza A - H1N1. São Paulo, 2009.

Disponível:

http://www1.saude.rs.gov.br/dados/1309185740669Recomenda\%E7\%F5es\%20para\%20crian\%E7as\%20com\%20quadro\% 20grave\%20de\%20Influenza\%20A\%20H1N1\%20-\%20Protocolo\%20AMIB\%20e\%20SBP.pdf

8- Frota RG, Moraes CEB, Muniz EB, Pereira NA, Vasconcelos BB, Oliveira MAS. Análise do boletim de Apgar em dados do Sistema de Informação sobre Nascidos Vivos registrados em um hospital do interior do estado do Ceará, Brasil. Revista de Medicina e Saúde de Brasília. 2016, 5(2). Disponível:

https://portalrevistas.ucb.br/index.php/rmsbr/article/view/6677/4563 
9- Figueiro-Filho EA, Ferreira CM, Oliveira VM, Silva VM, Kanomata LB, Tinos AL. Variáveis perinatais e associação de recém-nascidos de muito baixo peso ao nascer em hospital público universitário do Brasil. Revista Brasileira de Ginecologia e Obstetrícia. 2014, 36(1): 10-16. Disponível: http://www.scielo.br/pdf/rbgo/v36n1/0100-7203-rbgo-36-0100010.pdf

10- Kilsztajn S, Lopes ES, Carmo MSN, Reyes AMA. Vitalidade do recém-nascido por tipo de parto no Estado de São Paulo, Brasil. Cadernos de Saúde Pública. 2007, 23: 1886-1892. Disponível: http://www.scielo.br/pdf/csp/v23n8/15.pdf 11- Santana FG, Santos FS, Feitosa MO, Farias FBB, Santos FCS, Neto MS, Santos LH. Relação entre a idade materna e condições perinatais no município de Augustinópolis-TO. Revista de Pesquisa em Saúde. 2010, 11(3).

Disponível:http://www.periodicoseletronicos.ufma.br/index.php/revistahuufma/article/viewFile/782/496

12- Salem KB, Mhamdi SE, Amor IB, Sriha A, Letaief M, Soltani MS. Caracteristiques epidemiologiques et chronologiques des parturientes aux ages extremes dans la región de Monastir entre 1994-2003. Santé Publique. 2010, 88(8): 563-568.

Disponível:

https://www.researchgate.net/profile/Belguith_Sriha_Asma/publication/45651855_Epidemiological_and_chronological_ profile_of_the_parturientes_in_the_extreme_ages_in_the_Monastir_region_between_1994_and_2003/links/58811294 4585150dde3f3d9e/Epidemiological-and-chronological-profile-of-the-parturientes-in-the-extreme-ages-in-the-Monastirregion-between-1994-and-2003.pdf

13- Aquino MMA, Cecatti JG, Faundes A, Surita FGC. O Impacto da Idade Materna Avançada sobre os Resultados da Gravidez. Revista Brasileira de Ginecologia e Obstetrícia. 1998, 20(7): 389-394. Disponível:

http://www.scielo.br/pdf/rbgo/v20n7/a04v20n7.pdf

14- Moreira MDS, Gaiva MAM, Bitencourt RM. Mortalidade neonatal: características assistenciais e biológicas dos recémnascidos e de suas mães. Cogitare Enfermagem. 2012, 17(1). Disponível:

https://revistas.ufpr.br/cogitare/article/view/26383/17576

15- Secretaria de Estado da Saúde de São Paulo. Atenção à gestante e à puérpera no SUS-SP: manual técnico do pré-natal e puerpério. In: Atenção à gestante e à puérpera no SUS-SP: manual técnico do pré-natal e puerpério. 2010: $234-234$.

Disponível: http://www.saude.sp.gov.br/resources/ses/perfil/gestor/destaques/atencao-a-gestante-e-a-puerpera-no-sus$\mathrm{sp} /$ manual-tecnico-do-pre-natal-e-puerperio/manual_tecnicoii.pdf

16- Matias A, Montenegro N, Tiago P. Cálculo da Idade Gestacional Métodos e Problemas. Acta Médica Portuguesa. 2002, 15(1): 17-21. Disponível:https://www.actamedicaportuguesa.com/revista/index.php/amp/article/viewFile/1912/1480 17- Franciotti DL, Mayer GN, Cancelier ACL. Fatores de risco para baixo peso ao nascer: um estudo de caso-controle. Arquivos Catarinenses de Medicina. 2010, 39(3). Disponível: http://www.acm.org.br/revista/pdf/artigos/818.pdf 18- Lansky S, França E, Leal MC. Mortalidade perinatal e evitabilidade: revisão da literatura. Revista de Saúde Pública. 2002, 36: 759-772. Disponível: http://www.scielo.br/pdf/rsp/v36n6/13534.pdf 